\title{
REPARTO MODAL EN LAS INTERROGATIVAS EN EL ESTILO INDIRECTO LATINO
}

\begin{abstract}
The aim of this paper is to typify the modal distribution of the interrogative sentences studied here. The modal distribution proposed, after having been examined many texts, is mainly based on the distinction between the logical, impressive and expressive uses of the language, pointing out that those uses can appear and be hidden under an expression formally interrogative.
\end{abstract}

La gramática histórica se planteó el tema del empleo de los modos en las interrogativas del estilo indirecto e intentó dar diversas soluciones sin llegar a describir de un modo convincente la razón determinante del empleo del infinitivo o subjuntivo en cada uno de los casos.

Riemann llegó a decir, como síntesis del estado de la cuestión, que los gramáticos estaban lejos de ponerse de acuerdo sobre la regla que conviene dar. El mismo Riemann ${ }^{1}$ intentó también buscar esta regla, pero sus conclusiones van más en la línea de aportar datos sobre la frecuencia del empleo del infinitivo o subjuntivo que en la de dar con la clave que justifique el uso de una $u$ otra forma.

Los estudios realizados desde una perspectiva estructuralista sobre el empleo de los modos verbales por Mariner ${ }^{2}$, García Calvo ${ }^{3}$ y Rubio ${ }^{4}$ han clarificado decisivamente el uso de los modos en las oraciones independientes y subordinadas fuera del estilo indirecto; en cuanto al estilo indirecto se ha llegado a tipificar con claridad el uso de los

1 O. Riemann, «Les propositions interrogatives dans le style indirect en latin*, $R P h 7,1883$, pp. 112-131 y $164-169$.

2 S. Mariner, «Estructura de la categoría verbal modo en latín clásico», EMERITA 25, 1957, pp. 449-486. «Noción básica de los modos en el estilo indirecto», Barkita 33, 1965, pp. $47-59$.

3 A. García Calvo, «Preparación a un estudio orgánico de los modos verbales sobre el ejemplo del griego antiguos, BMeriTa 28,1960 , pp. 147.

4 L. Rubio, Introducción a la Sintaxis Estructural del Latín, II, Barcelona, 1976. 
modos, con la sola excepción de las oraciones interrogativas. En este último tipo de oraciones el reparto modal se realiza de una forma al parecer arbitraria, o según unos determinantes todavía no tipificados o tipificados solamente a nivel de tendencia ${ }^{5}$.

Con el afán de poner algo de luz en esta confusa situación, he estudiado numerosos textos en los que aparecen oraciones interrogativas en estilo indirecto y creo que, después de este análisis, puedo proponer una hipótesis que clarifique la razón determinante del empleo de un modo $\mathrm{u}$ otro.

Los textos estudiados corresponden a César, Livio y Tácito. Lógicamente una verificación total de la hipótesis sólo puede realizarse con el despojo exhaustivo de los textos de los autores estudiados, y aun así el alcance de la hipótesis afectaría solamente a estos autores. Debo confesar que este trabajo exhaustivo no lo he realizado, pero sí creo que los textos estudiados suponen, por su número, un muestreo suficientemente significativo como para poder emitir una hipótesis fundamentada.

Para ir caminando hacia la delimitación de este empleo, me parece necesario hacer una primera matización. Ciertamente, como dice Rubio ${ }^{6}$, la modalidad interrogativa de la frase queda en el estilo indirecto positivamente marcada, frente a las otras modalidades, por la entonación, pero, a mi juicio, este hecho no determina que el modo verbal sea en ellas irrelevante. Sería irrelevante si en efecto la entonación interrogativa fuera siempre y solamente el significante de la modalidad interrogativa. Pero la situación es bastante más compleja, ya que la entonación interrogativa puede ser a veces (y casi me atrevería a decir que la mayoría de las veces) el significante de un valor puramente estilístico. Es decir, puede suceder que la entonación interrogativa no corresponda de hecho a un estado de duda real en el hablante, o al menos no lo signifique prioritariamente, sino que sea el ropaje estilístico con el que se expresan otras actitudes.

La gramática tradicional distinguía las interrogativas retóricas de las interrogativas propiamente dichas, en cuanto que las primeras no solicitaban una respuesta del oyente ni suponían una duda real en el hablante. Creo que esta distinción no basta para desentrañar el complejo mundo lingǘstico que puede esconderse detrás de una expresión con entonación interrogativa. $\mathrm{Y}$ es precisamente el desentrañamiento

5 C. Fernández Martínez, «Las oraciones interrogativas en el estilo indirecto latino", Habis 12, 1981, pp. 81-87.

6 L. Rubio, op. cit., p. 71. 
de este complejo mundo lingüístico lo que me parece que puede conducir a la clarificación del reparto modal.

En otras palabras, considero necesario en primer lugar detectar de qué naturaleza es el mensaje que se está transmitiendo bajo la forma de una pregunta o, lo que es lo mismo, cuál es la intención predominante en el hablante al transmitir este mensaje.

Hay por tanto que distinguir en primer lugar lo que corresponde al contenido semántico de un mensaje y lo que se reduce a una mera cuestión de estilo. A la luz de esta distinción podemos decir que una oración interrogativa puede ser:

a) Semántica y estilísticamente interrogativa.

b) Semánticamente declarativa y estilísticamente interrogativa.

c) Semánticamente impresiva y estilísticamente interrogativa.

d) Semánticamente expresiva y estilísticamente interrogativa.

Es decir, bajo la entonación interrogativa el hablante puede estar realmente:

a) Preguntando.

b) Afirmando o negando algo.

c) Ejerciendo una influencia voluntaria sobre el oyente con la expresión de un mandato o deseo.

d) Expresando predominantemente un estado interior de ánimo.

Los casos $a$ y $b$ corresponden a un uso lógico del lenguaje, ya que, desde la actitud de duda, el hablante busca predominantemente la verdad, y desde la aseveración se afirma o se niega la verdad. El caso $c$ corresponde a un uso impresivo y el $d$ a un uso expresivo o predominantemente expresivo en el que el hablante puede expresar una situación de extrañeza, indignación, repulsa, etc.

Sólo en la situación a la entonación interrogativa tiene el valor de significante lingüístico correspondiente a un significado interrogativo. En las situaciones $b, c$ y $d$ la entonación interrogativa tiene simplemente un valor estilístico de refuerzo expresivo o atenuación cortés del mensaje impresivo.

Para ilustrar estas posibilidades, basten los siguientes ejemplos:

Cicerón, ad Att. I 14, 3.

Intellexi hominem (Pompeium) moueri: utrum Crassum inire eam gratiam quam ipse praetermississet, an esse tantas res nostras quae tam libenti senatu laudarentur? «Comprendí que Pompeyo vacilaba preguntándose si Craso pretendía atraerse la admiración... o si lo que yo habia hecho era tan importante como para que fuese agradable al senado.» 
Es ésta una pregunta real, Pompeyo delibera ante una doble alternativa. La frase es semántica y estilísticamente interrogativa.

Tácito, Ann. III 34.

Valerius. Messalinus... respondit... bella plane accintis obeunda, sed reuertentibus post laborem quod honestius quam uxorium leuamentum? «Valerio Mesalino respondió que a la guerra hay que ir sin embarazos, pero a la vuelta de los trabajos de ella, ¿qué hay más honesto que la compañf́a de la propia mujer?

Valerio afirma claramente que no hay nada más honesto que esto, por tanto, la frase es semánticamente aseverativa y estilísticamente interrogativa.

Livio, IV 43, 11-12.

Lucius Papillus Mugillanus castigando (dicebat): ... Quin illi remittendo de summa quisque iuris, mediis copularent concordiam...? « ¿Por qué no llegar a la concordancia en un punto medio, cediendo cada uno de sus posiciones extremas?

Lucio Papirio no está preguntando sobre las causas que impiden la concordia, sino que está exhortando a los dos bandos en litigio (senadores y tribunos de la plebe) a llegar a un acuerdo. La frase aquí es semánticamente impresiva y estilísticamente interrogativa: Quin copularent $=$ copulate. $\mathrm{El}$ efecto de la interrogación es de atenuación cortés de la fuerza de una invitación.

César, B. G. I 44, 7.

Ariouistus ad postulata Caesaris pauca respondit, de suis uirtutibus multa predicauit: ... Quid sibi uellet cur in suas possessiones ueniret? Prouinciam suam hanc esse Galliam sicut illam nostram.

Ariovisto no se pregunta por los motivos que César puede tener para venir a sus posesiones (bien claramente los conoce), la pregunta que realiza tiene un contenido semántico de expresión de su indignación por esta venida, algo equivalente a una exclamación indignada que podría equivaler a: ¡lo que va a querer!, ¡para qué va a venir! Evidentemente las intenciones de César son claras, como más adelante afirma el mismo Ariovisto: debere se suspicari simulata Caesarem amicitia, quod exercitum in Gallia habeat, sui opprimendi causa habere.

Como acabamos de ver, las distintas posibilidades semánticas que ofrece la entonación interrogativa pueden ponernos en la pista de las razones que determinan el uso del infinitivo o el subjuntivo. 
Conviene recordar aquí que el reparto de los modos en las oraciones no interrogativas del estilo indirecto se realiza de la siguiente forma: los usos lógicos quedan reservados al infinitivo en las oraciones no subordinadas, los usos impresivos y expresivos al subjuntivo.

En cuanto a las interrogativas de estilo indirecto, teniendo en cuenta todo lo dicho anteriormente, creo que el uso de los modos se puede sistematizar de una forma bastante similar: el subjuntivo quedaría reservado para los usos impresivos y predominantemente expresivos, y el infinitivo para los usos lógicos (oraciones semánticamente aseverativas e interrogativas).

Pero además el infinitivo puede emplearse como término no caracterizado para unos y otros, y este empleo está justificado por la naturaleza misma del infinitivo y por la peculiar situación que se produce en el estilo indirecto. Pues, en el estilo indirecto, al transmitir el autor de una obra el pensamiento y palabras de otra persona, puede querer matizar las distintas actitudes o estados de ánimo de su hablante, o bien desentenderse de ellas. Si se desentiende de ellas porque las considera irrelevantes, es el infinitivo el modo propio para expresar una noción en cierta manera abstraída de las matizaciones que su sujeto (el hablante) le aportaría. La irrelevancia puede proceder del hecho de que el contexto aporte suficiente información para matizar las actitudes del hablante.

Sintetizando lo dicho hasta ahora el reparto modal sería el siguiente:
I. Subjuntivo:
1) usos impresivos;
2) usos expresivos o predominantemente expresivos.
II. Infinitivo:
1) usos lógicos; 2) término no caracterizado, que puede incluir los usos
impresivos y expresivos.

Los textos que citaré a continuación ilustrarán las diversas posibilldades de empleo. Me voy a extender especialmente en los ejemplos ilustrativos del uso del subjuntivo por ser éste el término caracterizado de la oposición:

\section{INTERROGATIVAS EN SUBJUNTIVO}

1) Usos impresivos:

Livio, III 61, 14.

Nec cessabant Sabini... lacessere atque instare rogitantes... Quin illi congrederentur acie inclinandamque semel Fortunae rem darent? « ¿Por qué no librar la batalla y dejar que la Fortuna decida por una vez?*

Id., IV 43, 11-12 (cf. supra). 
Tácito, Ann. XIII 49.

Quin de bello aut pace, de uectigalibus et legibus, quibusque aliis res Romana contineretur, suaderet dissuaderetue?

En todos estos casos el hablante está expresando una invitación o una exhortación a otros para que actúen en un sentido determinado. Bajo la forma quin no subyace la indagación por los motivos de una actuación, sino una exhortación o mandato.

\section{2) Usos expresivos:}

En estos usos el hablante tampoco expresa un estado de duda, o al menos no lo expresa prioritariamente. El estado de duda se caracteriza por una suspensión del ánimo ante una decisión o un hecho. Pero en los casos a los que voy a aludir no hay un estado de suspensión de ánimo, sino que el hablante ha deliberado previamente sobre la razón o sinrazón de los hechos, o los ha intuido y expresa su situación o su toma de postura ante estos hechos. Esta situación puede ser de extrañeza ante lo ilógico o inesperado, indignación o repudio de un hecho, desaliento, etc.

Los ejemplos que voy a citar expresan esta variedad de situaciones:

César, B. G. I 40.

Si (Ariouistus) furore impulsus bellum intulisset, quid tandem uererentur? aut cur de sua uirtute aut de ipsius diligentia desperarent? «Si Ariovisto hubiera promovido la guerra impulsado por su furor, ¿qué tenían que temer o por qué desesperaban de su propio valor y diligencia?»

Con las interrogaciones que César dirige a sus soldados les está expresando su propia extrañeza y les está invitando a no temer ni desesperar. Se fusionan aquí el campo de lo impresivo y de lo expresivo, de tal manera que el contenido semántico podría ser el equivalente a: «me extraña que temáis, no temáis», "me extraña que desesperéis, no desesperéis».

Otro tanto podría decirse de ejemplos tales como:

Id., $i b$. IV 16, 4.

Si se inuito Germanos in Galliam transire non aequum existimaret, cur sui quicquam esse imperii aut potestatis trans Rhenum postularet?

Cur postularet podría igualmente interpretarse como: « ¿Cómo es posible que pidas?, no pidas». 
En el mismo sentido cabe interpretar textos tales como:

Id. B. C. 1, 32, 3 .

Qui si improbasset, cur ferri passus esset? Et si probasset, cur se uti populi beneficio prohibuisset?

Tácito, Ann. 13, 49.

Cur enim, si rem publicam egere libertate senatoria crederet, tam leuia consectaretur?

En todos estos ejemplos, con construcciones sintácticas idénticas, se propone una condición, cumplida la cual, se hace evidente la ilogicidad de lo que se pregunta y de aqui se desprende la necesidad de actuar de otra forma.

En otras ocasiones se dan una serie de razones previas que evidencian también la ilogicidad de lo que se pregunta, con lo cual la pregunta se convierte en una invitación a otra forma de actuación. Basten ejemplos tales como:

César, B. G. I 40, 2.

Ariouistus se consule cupidissime populi Romani amicitiam adpetisse: cur hunc tam temere quisquam ab officio discessurum iudicaret?

Id., B. G. I 72, 2.

Caesar in eam spem uenerat, se sine pugna rem conficere posse; cur etiam secundo proelio aliquos ex suis amitteret? cur uulnerari fateretur optime de se meritos milites? cur denique fortunam periclitaretur?

De hecho en ninguno de estos textos las preguntas responden a una situación real de duda o incertidumbre, sino que son expresivas de la postura ante los hechos de los distintos hablantes, postura de extraneza ante la ilogicidad de las distintas actuaciones.

Los textos que estudiaremos a continuación son expresivos de situaciones de indignación, repulsa o temor por parte de los hablantes:

Livio, VI 36, 11.

Sextius Liciniusque... artifices iam tot annorum usu tractandi animos plebis, primores patrum fatigabant: auderentne postulare ut... ipsis plus quingenta iugera habere liceret?... an placeret fenore circumuentam plebem... corpus in neruum ac supplicia dare? «Sexto y Licinio, expertos desde hacía años en manejar los ánimos de la plebe... fatigaban también a los más ilustres patricios preguntándoles: ¿Se atreverian a pedir para ellos más de cien yugadas? ¿Les gustaría que la plebe, agobiada por la usura, entregara su cuerpo al suplicio?»

LIII, $10^{\circ}-8$ 
Auderent y placeret tienen un valor semántico equivalente a: «¿Cómo es posible que se atrevan?», « ¿Cómo es posible que les guste?», « ies indignante! ». Por otra parte, el valor expresivo e impresivo están nuevamente fusionados; en concreto el impresivo está sugerido además contextualmente por la expresión: artifices tractandi animos plebis y por fatigabant. Sextio y Licinio, expresando su indignación, pretenden manipular a los patricios del mismo modo que manipulaban a la plebe para que no se atrevan a reclamar sus posesiones: auderent y placeret equivalen también a: "que no se atrevan», "que no les complazca».

En el ejemplo que voy a citar a continuación se puede ver el uso de un subjuntivo con valor expresivo coexistiendo con el uso de un infinitivo como término no caracterizado, también con valor expresivo, denotando ambos una situación de indignación. La posibilidad de emplear tanto uno como otro modo permite al autor una uariatio que resulta rentable estilísticamente:

Livio, VI 17, 4.

Audiebantur itaque propalam uoces exprobantium multitudini: ... Non obuersatam esse memoriam noctis illius, quae pene ultima atque aeterna nomini Romano fuerit? Non speciem agminis Gallorum, per Tarpeiam rupem scandendis? non ipsius $M$. Manlii qualem eum armatum, plenum sudoris ac sanguinis, ipso pene loue erepto ex hostium manibus uidissent? Selibrisne farris gratiam seruatori patriae relatam?

Non uidissent equivale semánticamente a: « ¿Cómo es posible que no hayan visto?». La indignación está al mismo tiempo sugerida contextualmente por exprobantium.

Igualmente expresivo de una situación de indignación es el siguiente texto de Livio:

\section{2, 9.}

Quidnam illi consules dictatores facturi essent, qui proconsulare imaginem tam saeuam ac trucem fecerint? Quidnam facturi essent? tiene un sentido equivalente a: « ¡lo que van a hacer!

Tácito, Hist. IV 62.

Medium omne tempus per uarias curas egere, ignauissimus quisque caesorum apud Vetera exemplo pauentes, melior pars rubore et infamia: Quale illud iter? Quis dux uia? \&En el intervalo estaban agitados por diversas inquietudes, los más débiles temblaban por la idea de una ma. sacre como en Vetera, los más valientes, llenos de rubor y vergüenza dećan: ¿Cuál va a ser el camino? ¿Quién podrá guiarlos en el recorrido?» 
Evidentemente los soldados no se preguntan cuál puede ser el camino o quién puede ser su jefe, porque ambas cosas las conocen, sino que expresan su situación de rubor, vergüenza e infamia: « ¡lo que va a ser el camino! », « ¡nadie los puede guiar!

\section{Id., Ann. I 41.}

Gemitusque ac planctus etiam militum aures oraque aduertere. Progrediuntur contuberniis: quis ille flebilis sonus? quod tam triste? feminas inlustres, non centurionem ad tutelam, non militem, nihil imperatoriae uxoris... Pudor inde ac miseratio...

Tácito recoge aquí el momento en que Agripina, acompañada de un cortejo de mujeres, se despide de Germánico y abandona el campamento. Los soldados salen de sus tiendas al oír las lamentaciones; la pregunta que se formulan a continuación no es tal, ya que, al salir, todo el espectáculo se revela ante sus ojos: ¿Cómo es posible que esto suceda? Las interrogaciones quis ille flebilis sonus? quod tam triste? son la expresión de su estado de ánimo: pudor et miseratio.

El texto que cito a continuación puede parecer que plantea una serie de verdaderas preguntas:

Id., Hist. IV 69, 1 y 3.

Et Valentini animum laudabant, consilium Auspicis sequebantur... Deterruit plerosque prouinciarum aemulatio: quod bello caput? unde ius auspicium peteretur? quam si cuncta prouenissent, sedem imperio legerent?... taedio futurorum praesentis placuere.

Los galos, ante la alternativa de hacer la guerra al pueblo romano, propuesta suscitada por Valentino, y la de pedir la paz, moción defendida por Auspex, se hacen una serie de preguntas. Pero hay que tener en cuenta que estas preguntas no suponen una duda real, ya que la situación que provocaría esta duda real, la guerra, no se va a producir, puesto que ellos han decidido previamente pedir la paz: consilium Auspicis sequebantur. Las interrogaciones son más bien la expresión del temor y desconcierto que se produce en ellos al imaginarse una posible situación de guerra. Terror y desconcierto sugeridos por deterruit plerosque prouinciam aemulatio, y por taedio futurorum.

Muchos textos más podrían citarse, todos los cuales son susceptibles de interpretación en este sentido: el contenido semántico de todos ellos es predominantemente expresivo o impresivo. En ellos la actitud lógica de búsqueda de la verdad está prácticamente excluida. En todos estos textos el modo verbbal es el subjuntivo. 
Esta norma de uso modal se cumple con total regularidad en todos los textos que he estudiado, con una sola excepción que describiré a continuación.

Cuando la pregunta se realiza con un verbo de pensar o de creer el modo correspondiente siempre es el subjuntivo, y este subjuntivo no tiene necesariamente un valor impresivo o expresivo, sino que puede corresponder a la formulación de una verdadera pregunta. ¿Cómo explicar esta peculiaridad sin alterar la coherencia del reparto modal?

El hecho de que la pregunta se realice mediante un verbo de pensar o creer supone en el hablante el deseo, real o ficticio, de penetrar en la subjetividad del preguntado o de conocer su juicio; una pregunta del tipo: quid deinde facturum censerent? (Livio, 43,4 ) está indicando un contenido semántico equivalente a: según su juicio, según su opinión, ¿qué hay que hacer? Esta actitud de deseo de penetrar en la subjetividad del preguntado queda caracterizada tanto desde el punto de vista léxico (por el contenido semántico del verbo), como desde el punto de vista gramatical (por el empleo de un modo que, al no expresar la realidad objetiva, es el más apropiado para expresar lo subjetivo).

Podríamos decir que en este tipo de ejemplos el subjuntivo está lexicalizado.

En el siguiente texto podemos apreciar la formulación de una verdadera pregunta:

Livio, V 20, 2-3.

Litteras ad senatum missit deum inmortalium benignitate... Veios iam fore in potestate populi Romani quid de praeda faciendum censerent?

Por otra parte hay que tener en cuenta que no todas las preguntas que se formulan con un verbo de pensar o creer tienen un contenido semántico de sondeo de opinión de otra persona con la formulación de una pregunta real. Algunas de ellas tienen un valor puramente expresivo, y en este caso el subjuntivo está doblemente justificado.

El texto siguiente puede ser indicativo de la situación ahora descrita:

Id., III 72, 4.

Scaptione hoc, contionali seni, adsignaturos putarent finitimos populos?

Los cónsules expresan su indignación ante la multitud, que quiere atender al parecer de Escapción, y exclaman: « ¿Cómo es posible que 
piensen que...?». Este uso, claramente expresivo del estado de ánimo del hablante, se incluye en los usos del subjuntivo con valor expresivo, antes descritos.

\section{INTERROGATIVAS EN INFINITIVO}

1) Usos lógicos:

a) puramente interrogativos o predominantemente interrogativos:

Son muy escasas las oraciones que en el estilo indirecto suponen un estado de duda real en el hablante. En estos casos el que interroga lo hace verdaderamente para saber lo que se le responderá, o se propone a sí mismo una cuestión que le preocupa, deseando encontrar la respuesta. Por supuesto esta interrogación puede ser expresiva de un estado de ánimo concreto, pero predomina el carácter de duda e inseguridad, es decir, predomina el uso lógico del lenguaje. Es el infinitivo el modo propio de este tipo de mensajes, con la única excepción a que he aludido más arriba bajo el nombre de «subjuntivo lexicalizado».

Basten los siguientes ejemplos:

Cicerón, ad Att. I 14, 3 (cf. supra).

Livio, II 32,6 .

Pauor ingens in urbe metuque mutuo suspensu erant omnia. Timere patres resident in urbe plebem, incerti manere eam an abire mallent: quamdiu autem tranquilam multitudinem fore? Quid futurum deinde si quod.. bellum existat?

La semántica de incerti revela claramente que la pregunta formulada responde a una verdadera duda. Por supuesto que hay una situación de temor, como en otros textos antes analizados, pero este temor aún no ha conducido a una toma de postura, y las preguntas son verdaderas preguntas correspondientes a una verdadera situación de duda. Contextualmente esta duda está marcada por los términos: suspensa e incerti.

Tácito, Ann. II 70.

Quid deinde misserrimae coniugi euenturum?

Germánico, lleno de ansiedad, se pregunta por el futuro de su esposa. 
b) usos aseverativos:

Tácito, Ann. III 34, 46.

Valerius Messalinus... respondit... ipsorum magistratuum nonne plerosque uariis libidinibus obnoxios?... num ergo omnis caetibes integros?

Ambas preguntas tienen un contenido semántico aseverativo equivalente a: muchos de los mismos magistrados son responsables de diversos libertinajes, no todos los célibes son hombres íntegros.

\section{2) Infinitivo como término no caracterizado:}

Puede incluir los usos expresivos e impresivos.

Son especialmente elocuentes los ejemplos en que, dentro de un mismo contexto, se da la alternancia subjuntivo-infinitivo.

Recuérdese el texto citado supra: Livio, VI 17, 4. La misma alternancia encontramos en:

Id., III 72, 4.

Scaptione hoc, contionali seni, adsignaturos putarent finitimos populos? Clarum hoc fore imagine Scaptium; sed populum Romanum quadruplatoris et interceptoris litis alienae personam laturum. Quem enim hoc priuatae rei iudicem fecisse ut sibi controuersiam adiudicaret rem?

Las dos preguntas son claramente expresivas de la situación de indignación de los cónsules, no de una verdadera situación de duda.

\section{Ciriaca morano}

